\title{
Development of a biosensor platform for phenolic compounds using a transition ligand strategy
}

Lion Konstantin Flachbart ${ }^{1}$, Christoph Gerhard Wilhelm Gertzen ${ }^{2,3,4}$, Holger Gohlke ${ }^{2,3}$, and Jan Marienhagen ${ }^{1,5, *}$

${ }^{1}$ Institute of Bio- and Geosciences, IBG-1: Biotechnology, Forschungszentrum Jülich, D-52425 Jülich, Germany

${ }^{2}$ Institute for Pharmaceutical and Medicinal Chemistry, Heinrich Heine University Düsseldorf, Universitätsstr. 1, 40225 Düsseldorf, Germany

3John von Neumann Institute for Computing (NIC), Jülich Supercomputing Centre (JSC) and Institute of Biological Information Processing (IBI-7: Structural Biochemistry), Forschungszentrum Jülich $\mathrm{GmbH}, 52425$ Jülich, Germany

${ }^{4}$ Center for Structural Studies (CSS), Heinrich Heine University Düsseldorf, Universitätsstr. 1, 40225 Düsseldorf, Germany

${ }^{5}$ Institute of Biotechnology, RWTH Aachen University, Worringer Weg 3, D-52074 Aachen, Germany

* To whom correspondence should be addressed.

Tel: +49 246161 2843; Fax: +49 246161 2710; Email: j.marienhagen@fz-juelich.de 
Supporting Table S1. Strains and plasmids used in this study.

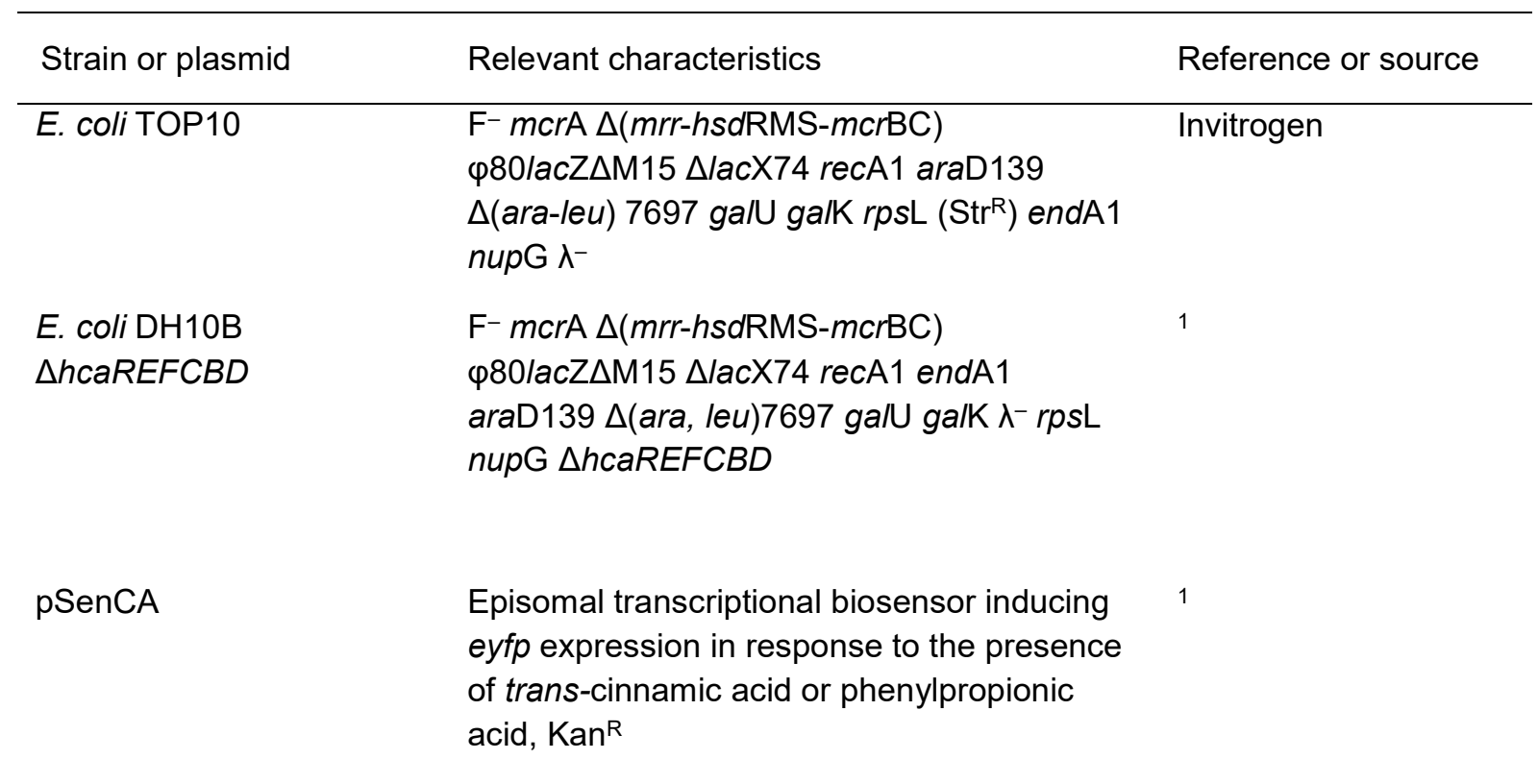

Supporting Table S2. Oligonucleotides used in this study. Underlined positions indicate degenerated codons.

\begin{tabular}{ll}
\hline Primer name & Sequence $\left(5^{\prime} \rightarrow 3^{\prime}\right)$ \\
\hline seq_hcaR_fw & CACACGGTCACACTGCTTCC \\
seq_hcaR_rv & TGAACAGCTCCTCGCCCTTG \\
amp_hcaR_fw & TGCCGTTACGCTTGCCAAACGTTC \\
amp_hcaR_rv & GAACTACGGCATTTACGCTATTTCGTCGCAG \\
amp_pSenCA_w/o_hcaR_fw & GTAAATGCCGTAGTTCCATCACCTTCCCCTTGTTATCGAAAAAACG \\
amp_pSenCA_w/o_hcaR_rv & GCAAGCGTAACGGCATAAGAGCTCCCGGGCCCG \\
hcaR-V97/P98-rv & GCAATAAATTCACTTCCGCCGAMNNMNNAAAGCCAATGGTTAA \\
& TTGTCTGTCTTCCTGAACAATTTTCCGC \\
hcaR-V97/P98-fw & GCGGAAAATTGTTCAGGAAGACAGACAATTAACCATTGGCTTTNNK \\
hNKTCGGCGGAAGTGAATTATTGC \\
hcaR-T128-rv & CGAATTTTTTCCTCCTGTTGCGTMNNGATTAAACTCACCAGCTCAA \\
hcaR-T128-fw & TCAAG \\
& CTTGATTGAGCTGGTGAGTTTAATCNNKACGCAACAGGAGGAAAA \\
hcaR-P195/V196/Y197-fw & AATTCG \\
& GGATGGCGTGAATTTCGTCAGTACCGATNNKNNKNNKTCCGGTTC \\
hcaR-P195/V196/Y197-rv & GCTTGCGCCGATCGTTAA \\
& TTAACGATCGGCGCAAGCGAACCGGAMNNMNNMNNATCGGTACT \\
& GACGAAATTCACGCCATCC
\end{tabular}




\begin{tabular}{|c|c|}
\hline hcaR-S198/G199/S200-fw & $\begin{array}{l}\text { GGCGTGAATTTCGTCAGTACCGATCCGGCGTATNNKNNKNNKCTT } \\
\text { GCGCCGATCGTTA }\end{array}$ \\
\hline hcaR-S198/G199/S200-rv & $\begin{array}{l}\text { TAACGATCGGCGCAAGMNNMNNMNNATACGCCGGATCGGTACTG } \\
\text { ACGAAATTCACGCC }\end{array}$ \\
\hline hcaR-L201-rv & GCTTTAACGATCGGCGCMNNCGAACCGGAATACGCCG \\
\hline hcaR-L201-fw & CGGCGTATTCCGGTTCGNNKGCGCCGATCGTTAAAGC \\
\hline hcaR-E101/V102-rv & $\begin{array}{l}\text { CGAAACATCGGTAATACTTTTGGCAATAAATTMNNMNNCGCCGAT } \\
\text { GGCACAAAGCCAATGGTTAATTGTC }\end{array}$ \\
\hline hcaR-E101/V102-fw & $\begin{array}{l}\text { GACAATTAACCATTGGCTTTGTGCCATCGGCGNNKNNKAATTTATT } \\
\text { GCCAAAAGTATTACCGATGTTTCG }\end{array}$ \\
\hline hcaR-N224/I225/L226-rv & $\begin{array}{l}\text { CCCCATGCCCACCAGATTCATGGTCACMNNMNNMNNCGTTGCCA } \\
\text { CCTGGACGATATTTGGCTG }\end{array}$ \\
\hline hcaR-N224/I225/L226-fw & $\begin{array}{l}\text { CAGCCAAATATCGTCCAGGTGGCAACGNNKNNKNNKGTGACCATG } \\
\text { AATCTGGTGGGCATGGGG }\end{array}$ \\
\hline hcaR-S99/A100-rv & $\begin{array}{l}\text { CATCGGTAATACTTTTGGCAATAAATTCACTTCMNNMNNTGGCACA } \\
\text { AAGCCAATGGTTAATTGTCTGTCTTC }\end{array}$ \\
\hline hcaR-S99/A100-fw & $\begin{array}{l}\text { GAAGACAGACAATTAACCATTGGCTTTGTGCCANNKNNKGAAGTG } \\
\text { AATTTATTGCCAAAAGTATTACCGATG }\end{array}$ \\
\hline hcaR-V227/T228-rv & $\begin{array}{l}\text { CCAGCCCCATGCCCACCAGATTCATMNNMNNCAGAATATTCGTTG } \\
\text { CCACCTGGACG }\end{array}$ \\
\hline hcaR-V227/T228-fw & $\begin{array}{l}\text { CGTCCAGGTGGCAACGAATATTCTGNNKNNKATGAATCTGGTGGG } \\
\text { CATGGGGCTGG }\end{array}$ \\
\hline hcaR-M245/N246-rv & $\begin{array}{l}\text { CGAAAAACAACCTGCCCGGTATTAAAATTMNNMNNATAACCGGGT } \\
\text { ATCAAAGTGACGCCCAGCC }\end{array}$ \\
\hline hcaR-M245/N246-fw & $\begin{array}{l}\text { GGCTGGGCGTCACTTTGATACCCGGTTATNNKNNKAATTTTAATAC } \\
\text { CGGGCAGGTTGTTTTTCG }\end{array}$ \\
\hline hcaR_mutH101Y102-V97/P98-rv & $\begin{array}{l}\text { GGCAATAAATTATAATGCGCCGAMNNMNNAAAGCCAATGGTTAAT } \\
\text { TGTCTGTCTTCCTGAACAATTTTCCGCGC }\end{array}$ \\
\hline hcaR_mutH101Y102-V97/P98-fw & $\begin{array}{l}\text { GCGCGGAAAATTGTTCAGGAAGACAGACAATTAACCATTGGCTTTN } \\
\text { NKNNKTCGGCGCATTATAATTTATTGCC }\end{array}$ \\
\hline hcaR_mutH101Y102-S99/A100-rv & $\begin{array}{l}\text { AAACATCGGTAATACTTTTGGCAATAAATTATAATGMNNMNNTGGC } \\
\text { ACAAAGCCAATGGTTAATTGTCTGTCTTCCTG }\end{array}$ \\
\hline hcaR_mutH101Y102-S99/A100-fw & $\begin{array}{l}\text { CAGGAAGACAGACAATTAACCATTGGCTTTGTGCCANNKNNKCATT } \\
\text { ATAATTTATTGCCAAAAGTATTACCGATGTTT }\end{array}$ \\
\hline
\end{tabular}


Supporting Table S3. All residues in the HcaR ligand binding domain targeted by SSM and MSSM. Simultaneously targeted residues are listed together. Library size and completeness according to the GluelT algorithm for each library constructed from pSenCA and pSenGeneral are listed. ${ }^{2}$

\begin{tabular}{|ccc|cc|cc|}
\hline $\begin{array}{c}\text { Library } \\
\text { no. }\end{array}$ & Residue(s) & $\begin{array}{c}\text { Mutagenesis } \\
\text { step }\end{array}$ & $\begin{array}{c}\text { Library } \\
\text { size }\end{array}$ & $\begin{array}{c}\text { GluelT } \\
\text { completeness } \\
{[\%]}\end{array}$ & $\begin{array}{c}\text { pibrary } \\
\text { size }\end{array}$ & $\begin{array}{c}\text { GluelT } \\
\text { completeness } \\
\text { [\%] }\end{array}$ \\
\hline 1 & V97, P98 & 1 and 2 & 6,000 & 100 & 3,000 & 98 \\
2 & E101, V102 & 1 and 2 & 4,500 & 100 & - & - \\
3 & T128 & 1 and 2 & 100 & 98 & 200 & 100 \\
4 & P195, V196, Y197 & 1 and 2 & 57,000 & 95 & 55,000 & 95 \\
5 & S198, G199, S200 & 1 and 2 & 60,000 & 96 & 56,000 & 95 \\
6 & L201 & 1 and 2 & 400 & 100 & 180 & 99 \\
7 & N224, I225, L226 & 1 and 2 & 61,000 & 96 & 70,000 & 97 \\
\hline 8 & S99, A100 & 2 & 3,900 & 99 & 3,280 & 98 \\
9 & V227, T228 & 2 & 4,100 & 99 & 5,300 & 99 \\
10 & M245, N246 & 2 & 4,200 & 99 & 7,900 & 100 \\
\hline
\end{tabular}


Supporting Table S4. Mutants isolated in the screening towards a HcaR regulator with relaxed ligand specificity and fluorescence response of respective biosensor variants. The codon sequence and the encoded amino acid at positions 101 and 102 of the wild-type HcaR protein and six isolated variants are given. For comparison of the different biosensor variants, the respective fold induction in specific EYFP fluorescence in response to the presence of different ligands is shown. In contrast to other data presented in this manuscript, this cultivation was performed with an inoculum $\mathrm{OD}_{600}$ of 1 , and fluorescence measurements were performed $4 \mathrm{~h}$ after cultivation $\left(37^{\circ} \mathrm{C}\right)$. Abbreviations: $\mathrm{CA}$, trans-cinnamic acid; PP, phenylpropionic acid; 3HPP, 3-hydroxyphenylpropionic acid; 3HCA, 3-hydroxycinnamic acid 3,5-dihydroxyphenylpropionic acid (3,5DHPP).

\begin{tabular}{ccccc|ccccc}
\hline Variant & $\begin{array}{c}\text { Codon } \\
\mathbf{1 0 1}\end{array}$ & $\begin{array}{c}\text { Residue } \\
\mathbf{1 0 1}\end{array}$ & $\begin{array}{c}\text { Codon } \\
\mathbf{1 0 2}\end{array}$ & $\begin{array}{c}\text { Residue } \\
\mathbf{1 0 2}\end{array}$ & CA & PP & 3HPP & 3HCl & 3,5DHPP \\
\hline WT- & & & & & & & & & \\
HcaR & GAA & E & GTG & V & 45.7 & 43.4 & 2.0 & 2.5 & 1.9 \\
1 & TGT & C & TTT & F & 3.2 & 3.5 & 2.9 & 3.4 & 3.3 \\
2 & AGT & S & TTT & F & 4.8 & 5.1 & 4.2 & 5.0 & 7.7 \\
3 & GCT & A & TTT & F & 4.8 & 5.1 & 4.2 & 5.0 & 1.5 \\
4 & ACT & T & TTT & F & 5.3 & 6.8 & 4.9 & 5.4 & 9.3 \\
5 & ACG & T & TTT & F & 3.8 & 4.1 & 3.7 & 4.2 & 3.9 \\
6 & CAT & H & TAT & Y & 10.9 & 11.8 & 10.4 & 11.5 & 8.4 \\
\hline
\end{tabular}




\section{Structural consequences E101H and V102Y}
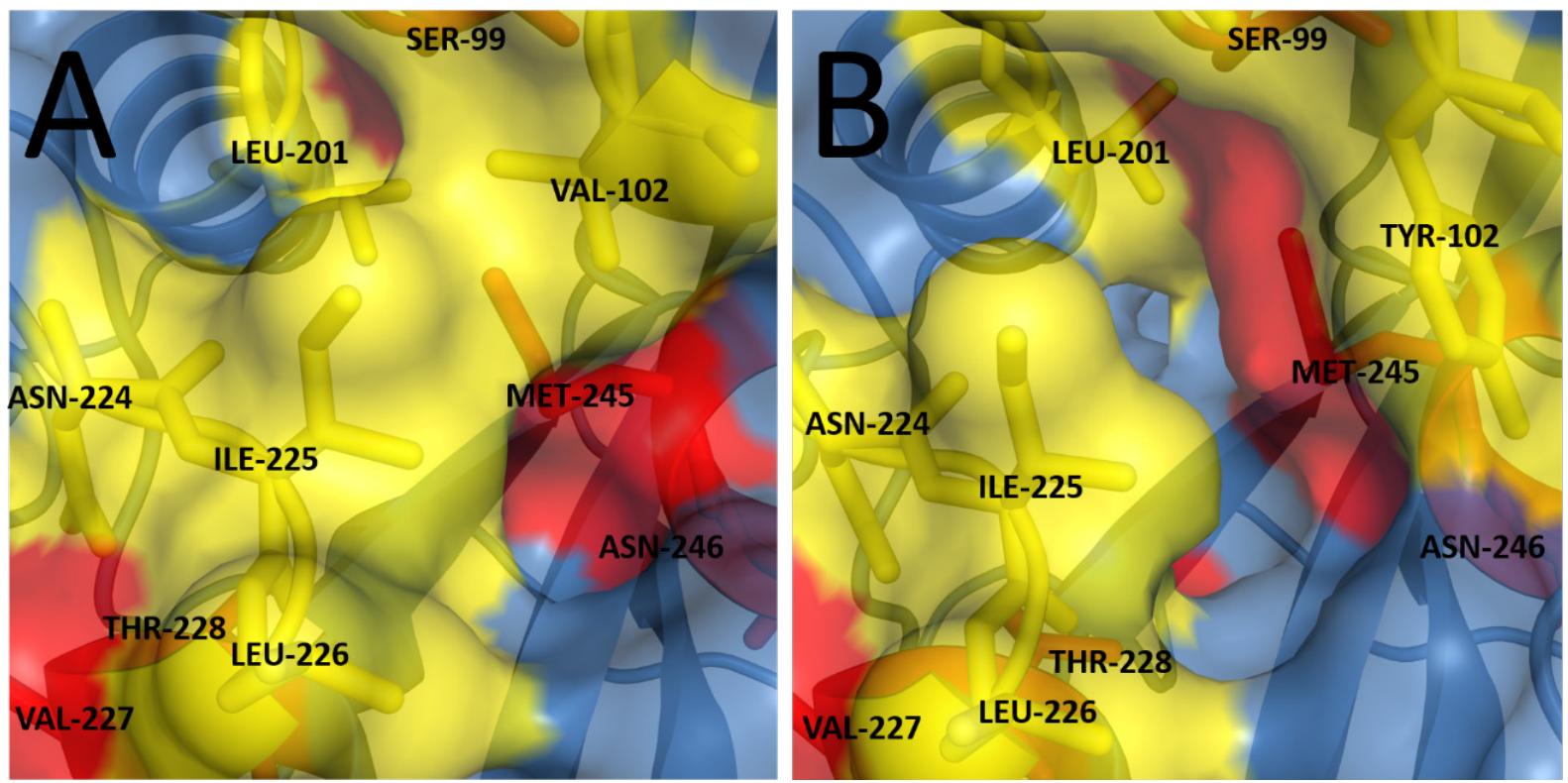

Supporting Figure S1. Structural consequences of amino acid substitutions E101H and V102Y. Figures show a close up of the HcaR binding cleft. Residues targeted in the first screening campaign are shown in yellow and additional targets of subsequent screenings in red. (A) Structure of HcaR. V102 and I225 are oriented towards each other, constraining the width of the binding cleft. (B) Structure of HcaR E101H/V102Y. By rotation of Y102, the binding cleft opens and M245 becomes accessible. 


\section{Second round of FACS-screening for the isolation of specific biosensors}

Initially, a positive FACS - enrichment step (FES) was performed (top $5 \%$ fluorescent cells), and 200,000 E. coli cells carrying a responsive biosensor variant were sorted directly into liquid medium. The sorted cells were cultivated in the presence and absence of the respective target ligand to quantify the proportion of constitutively fluorescent cells after the first enrichment step (Figure 3, FES 1). Roughly, $10 \%$ of these variants showed intermediate fluorescence in the absence of the respective target ligand and thus possessed an undesired phenotype. However, in the cultures where the respective target ligand was supplemented, most cells exhibited strong fluorescence.

To further enrich variants providing a strong biosensor response, another positive sorting step was performed, again with and without supplementation of the target ligands (Figure 3, FES 2). At this point, a strong fluorescence response without the addition of any target ligand was detected. To remove false-positive variants constitutively expressing eyfp, a negative FACS sorting step was included, in which the $5 \%$ of all cells exhibiting the lowest fluorescence were isolated (Figure 3, FES 3). To further enrich responsive biosensor variants, two additional FACS enrichment steps, one positive (upper $5 \%$, Figure S2, FES 4) and one negative (lower $55 \%$, Figure S2, FES 5), were performed. Upon recultivation, most of the remaining cells (> $80 \%$ ) in all campaigns showed a strong fluorescence response in the presence of the respective target ligand and a low fluorescence without target ligand supplementation. 


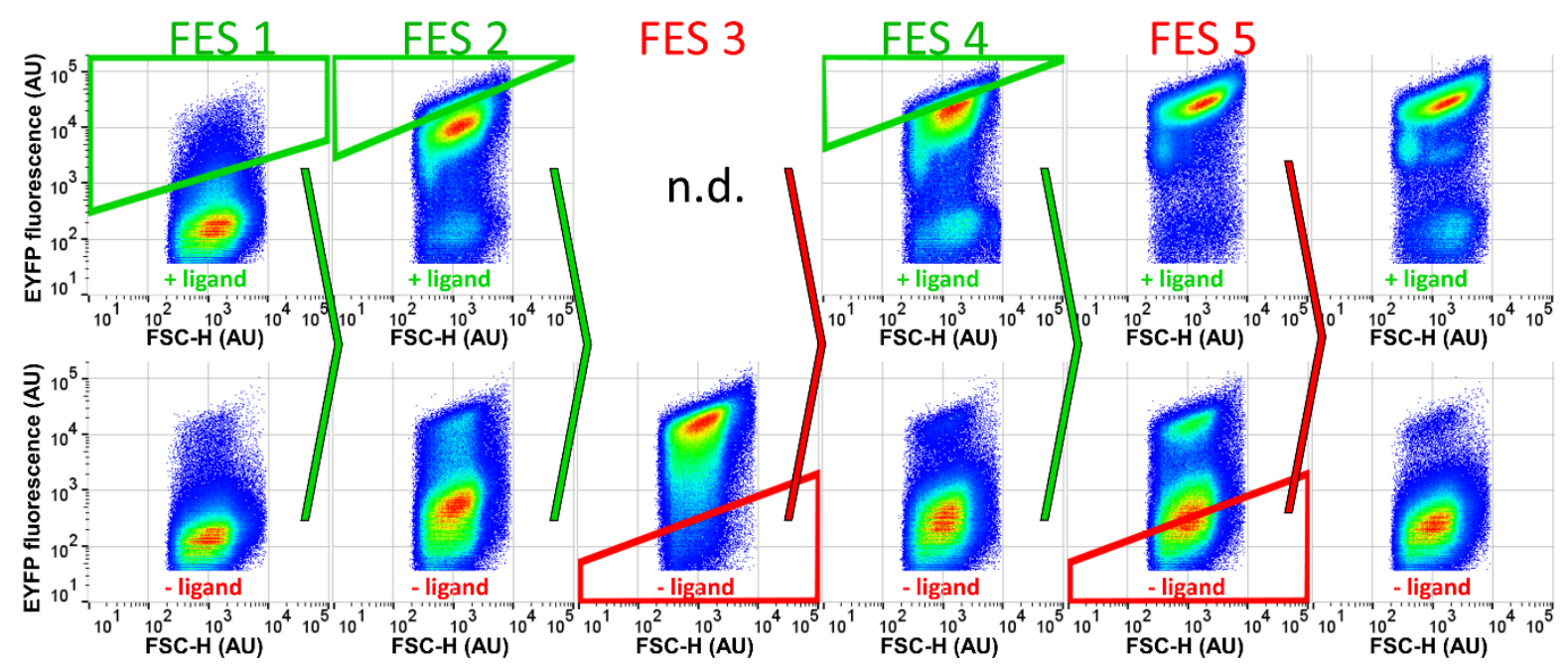

Supporting Figure S2. Second FACS-based screening of different hcaR libraries to isolate specific biosensor variants. Pseudocolor plots of representative data from the 6MSA FACS screening campaign comprised of five FACS-enrichment steps (FES). Both plots on the far left show the pooled, unsorted libraries. Each arrow depicts one round of FACS enrichment and recultivation. All cultivations were performed either with supplementation of $2 \mathrm{mM} 6 \mathrm{MSA}$ (upper diagrams) to check for a ligand-specific biosensor response, or without 6MSA (lower diagrams) to keep track of emerging false positive sensor variants. Green arrows indicate positive FACS sorting steps (top $5 \%$ fluorescent cells isolated) and red arrows negative FACS sorting steps (lower $5 \%$ of cells in FES 3 and lower $55 \%$ of cells in FES 5, respectively). For details the reader is referred to the text. Abbreviations: FSC-(H), Front scatter signal height; n.d., this cultivation was not performed. 

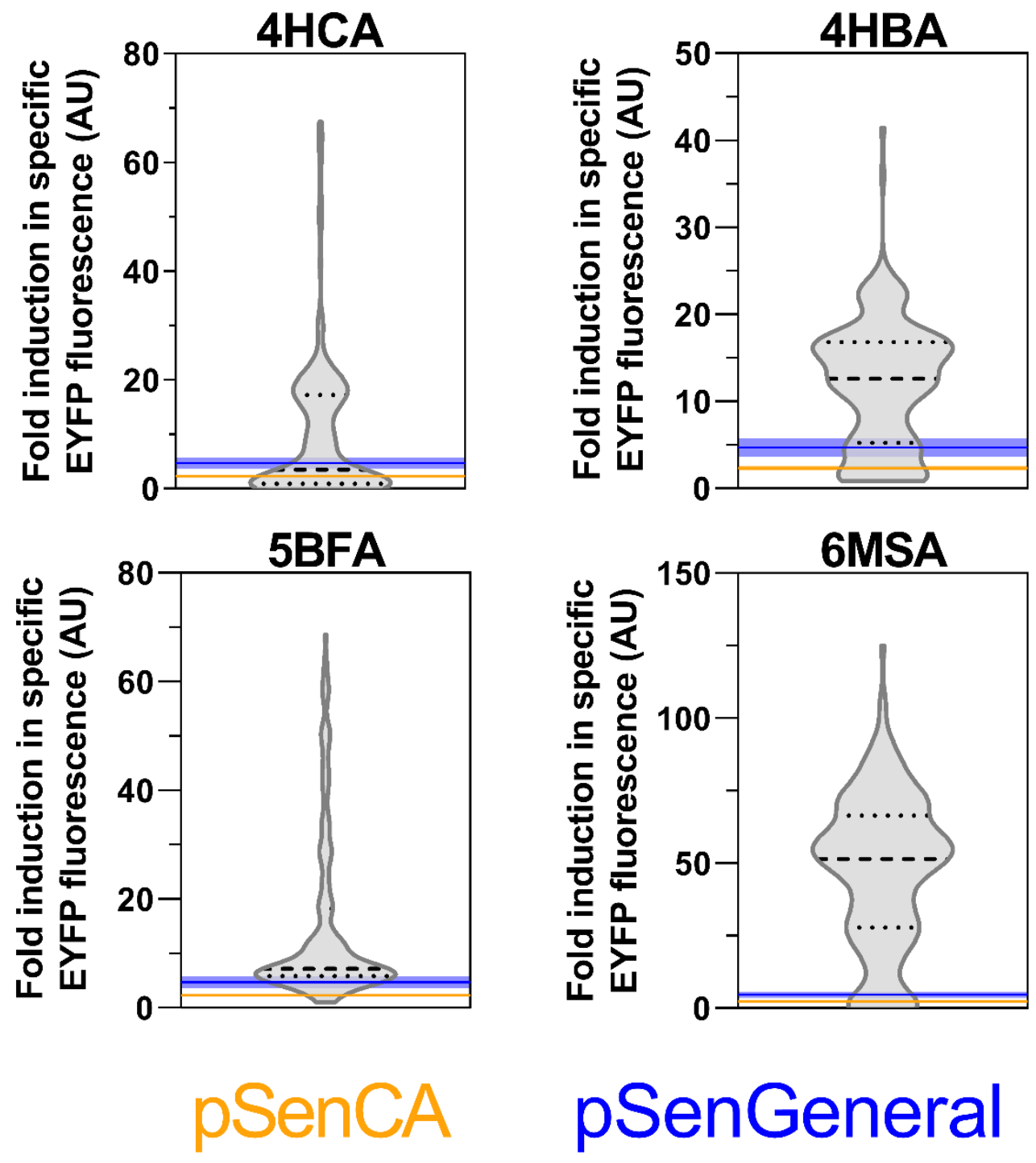

Supporting Figure S3. Fluorescence response of isolated biosensor variants to the respective target ligands. For each target ligand, 184 clones were isolated and individually characterized as to their fluorescence response. The distribution is shown as violin plots; dashed lines depict median, dotted lines depict quartiles of each distribution. Yellow and blue lines and shading depict fold induction and standard deviation of the benchmark biosensors Top10 pSenCA and pSenGeneral, respectively. Abbreviations: 4HCA, p-coumaric acid; 4HBA, 4-hydroxybenzoic acid; 5BFA, 5-bromoferulic acid; 6MSA, 6-methylsalicylic acid. 


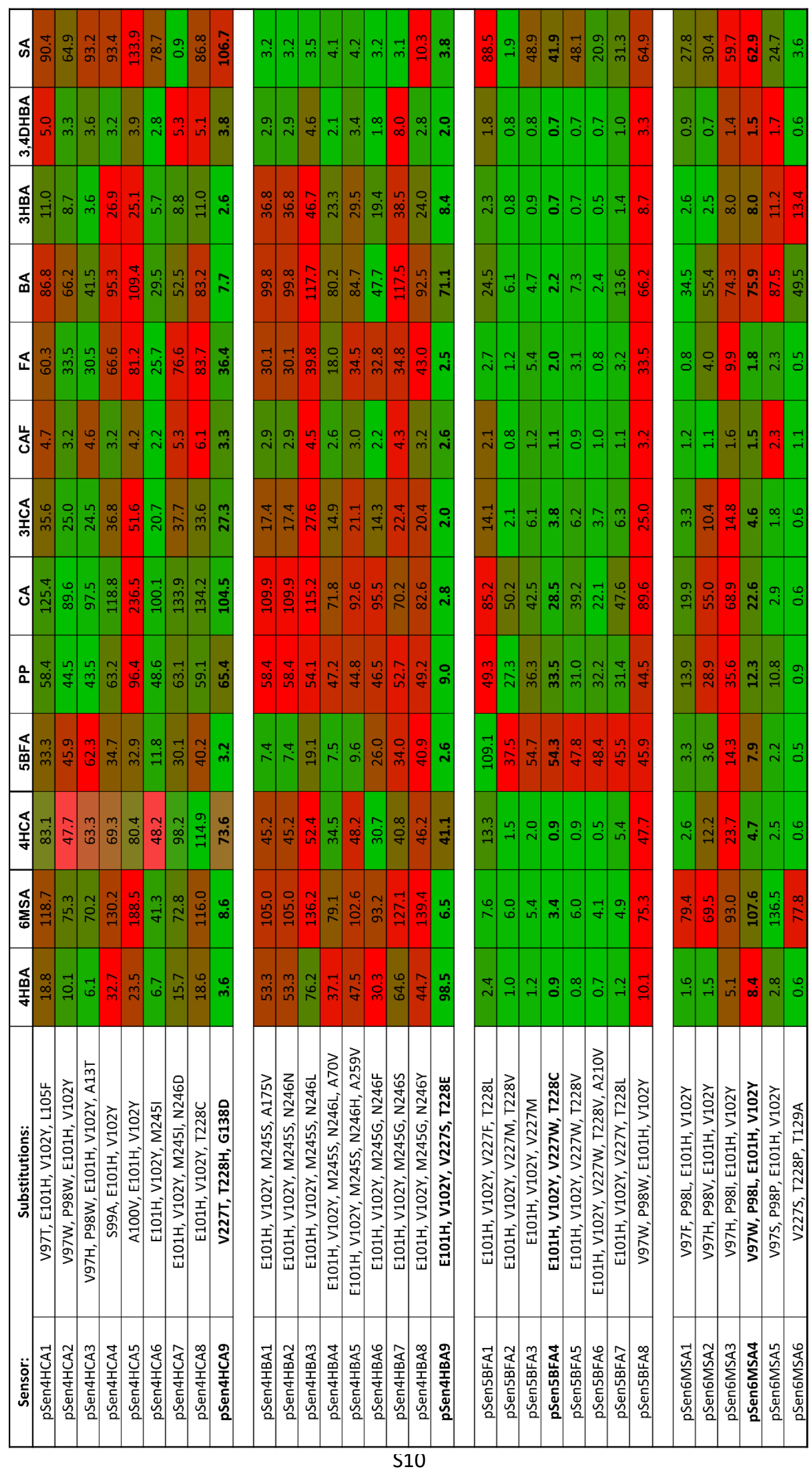


Supporting Table S5. Amino acid substitutions in the transcriptional regulator HcaR observed in the 31 sensor variants isolated in the second FACS screening. Response to the four target ligands 4HBA, 6MSA, 4HCA and 5BFA as well as the native ligands PP and CA and seven benchmark ligands is shown as fold induction in specific EYFP fluorescence. The response to each ligand is compared within the respective data set. Target ligands: The stronger the response to the target ligand, the greener the color of the respective box. Low biosensor responses are displayed in red. In case of all other ligands for the same biosensor, the lowest response is shown in green and increasing reponse is depicted by increasing red color. Responses of selected sensor variants in bold letters are compared in detail in figure 3 of the main text. Abbreviations: 4HBA, 4-hydroxybenzoic acid ; 6MSA, 6 methylsalicylic acid; 4HCA, p coumaric acid ; 5BFA, 5 bromoferulic acid ; PP, phenylpropionic acid; CA, transcinnamic acid; 3HCA, 3 hydroxycinnamic acid; CAF, caffeic acid; FA, ferulic acid; BA, benzoic acid; 3HBA, 3-hydroxybenzoic acid; 3,4DHBA, 3,4 dihydroxybenzoic acid; SA, salicylic acid. 


\section{Residual biosensor response of 96 isolated variants to PP and CA}

Although a small number (three and five mutants of the set of 86 , respectively) of the sensors exhibit only minimal $(<10 \%)$ residual fold induction in the presence of the native ligands PP and CA, the response was only slightly reduced in most of the isolated sensors (Figure S3). In order to eliminate variants with WT specificity during FACS, the native ligands will be used as decoys in future projects. That way, all variants induced by the decoy ligand can be removed from the library by sorting all variants that do not exhibit increased fluorescence under these conditions. Unfortunately, we could not perform such experiments. The E. coli strain used in this study carries a chromosomal copy of $h c a R^{W T}$ for high transformation efficiency for the construction of large libraries. This results in sufficient HcaR regulator to drive eyfp expression from a pSenCA plasmid deficient of the hcaR gene (data not shown).
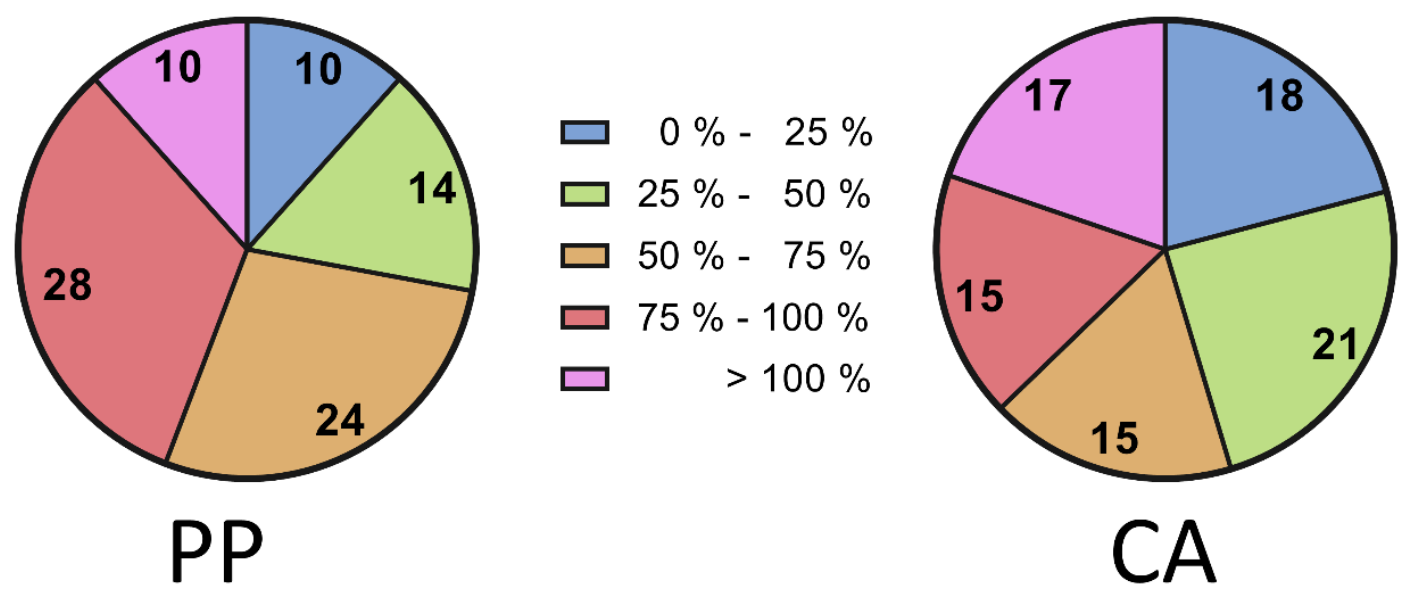

Supplementary Figure S4. Residual biosensor response of 96 isolated variants to PP and CA as native ligands of HcaR. Distribution of fold induction in specific EYFP fluorescence response is shown, relative to the response of pSenCA as depicted in the legend. The total number is 94 , as two $\mathrm{R} 37 \mathrm{H}$ mutants were not considered due to insufficient overall performance. Abbreviations: PP, phenylpropionic acid; CA, trans-cinnamic acid. 


\section{Heavy atom RMSD as a function of simulation time and per residue RMSF of four different HcaR variants}
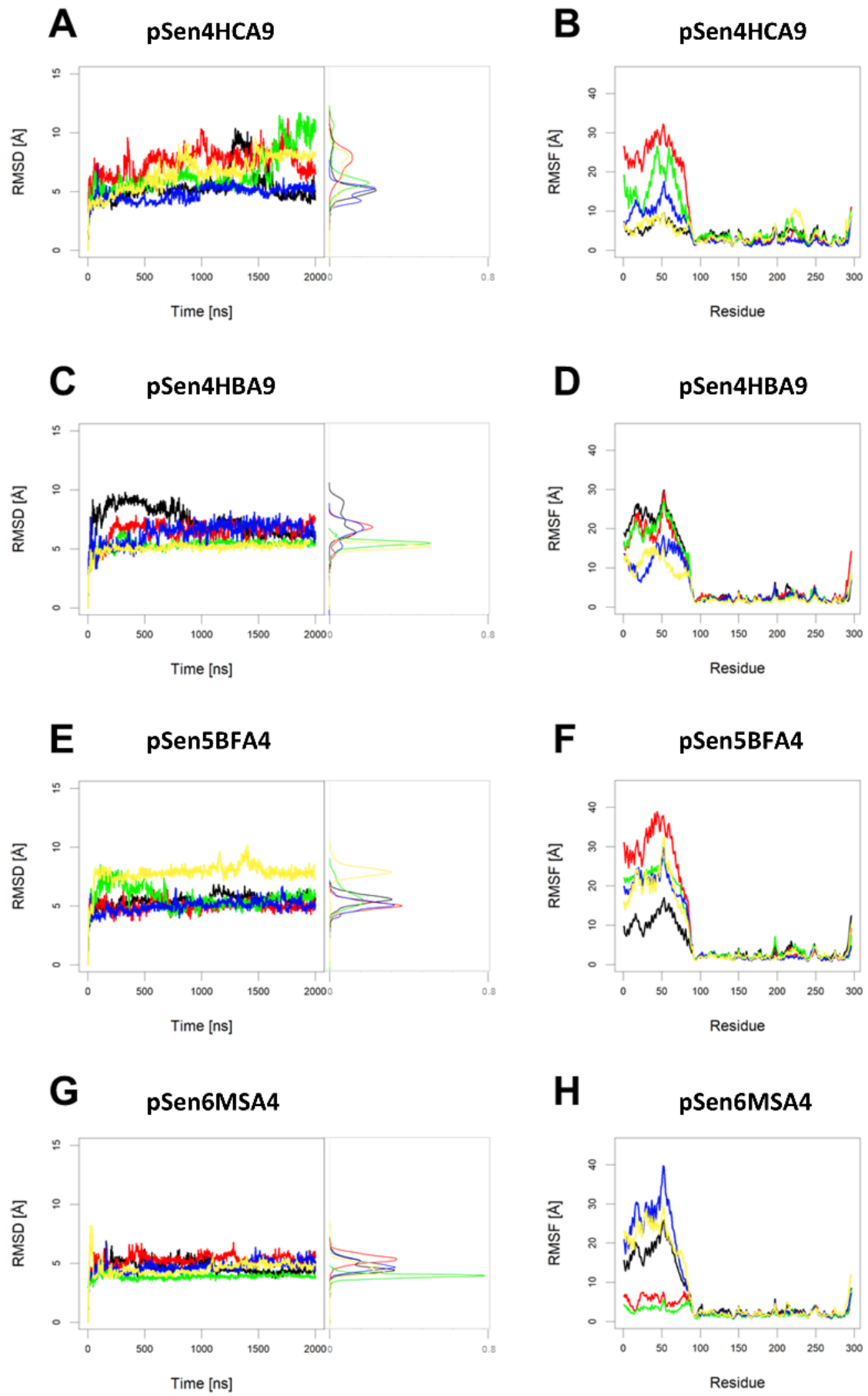

Supplementary Figure S5. Heavy atom RMSD as a function of simulation time and perresidue RMSF of four different HcaR variants from five MD simulations each. The heavy atom RMSD for HcaR of (A) pSen4HCA9, (C) pSen4HBA9, (E) pSen5BFA4, (G) pSen6MSA4 for residues 87-296, constituting the domains forming the binding pocket, are shown as time series and propensity distributions. The propensity distributions were normalized such that the area under the curves add up to 1. Per-residue RMSF for all residues of HcaR in (B) pSen4HCA9, (D) pSen4HBA9, (F) pSen5BFA4, (H) pSen6MSA4 after alignment on residues 87-296. The $\mathrm{N}$-terminal domain (residues 1-86) connected by a flexible linker to the ligand binding domains is mobile, which is reflected in RMSF values up to $41 \AA$. 


\section{Predicted binding modes of 4HBA and 4HCA in the HcaR variant of pSen4HBA}

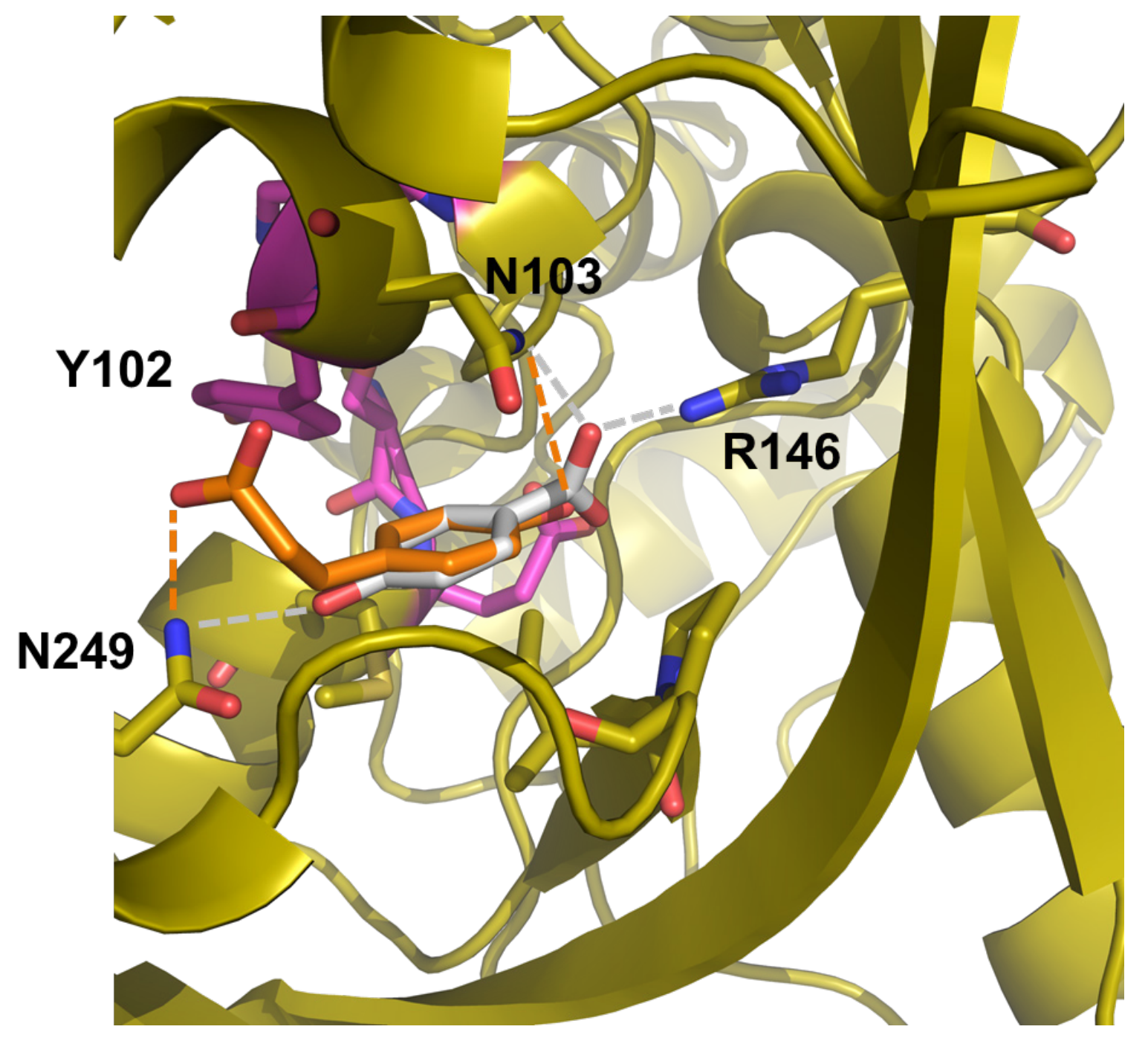

Supplementary Figure S6. Comparison of the predicted binding modes of 4HBA and 4HCA in the HcaR variant of pSen4HBA9. Comparison of the predicted binding modes of 4HBA (white) and 4HCA (orange) in HcaR (yellow). 4HCA forms a hydrogen bond to N103 with its hydroxyl group in position 4 of the phenyl ring and a hydrogen bond to N249 with its carboxylic acid moiety (orange dotted lines). 4HBA forms a hydrogen bond to N103 with its carboxylic acid moiety, a hydrogen bond to N249 with its hydroxyl group in position 4 of the phenyl ring, and a salt bridge with R146 with its carboxylic acid moiety (grey dotted lines). 


\section{Docking poses of ligands in the HcaR variants of pSen5BFA4 and pSen6MSA4}
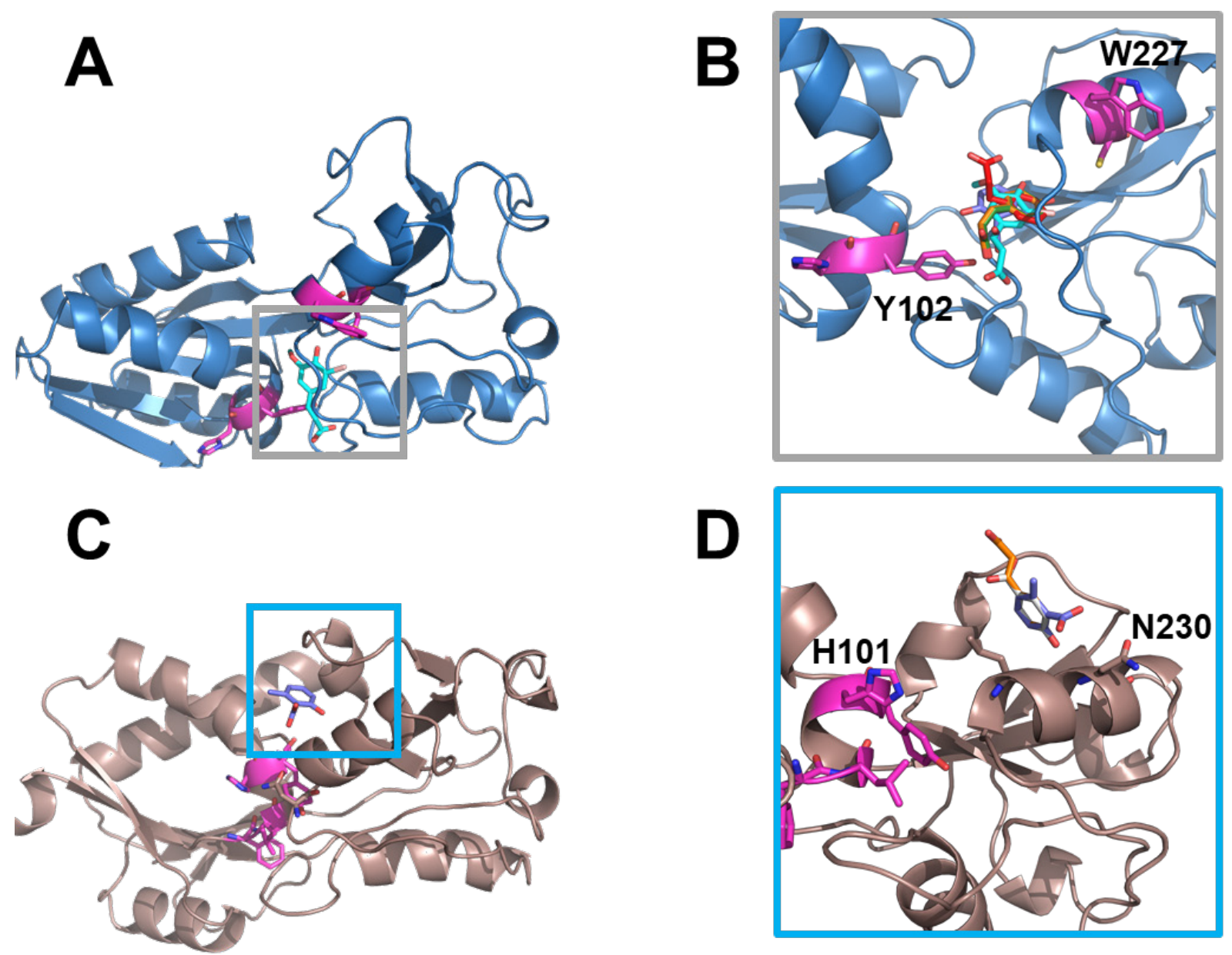

Supplementary Figure S7. Docking poses of ligands in the HcaR variants of pSen5BFA4 and pSen6MSA4. HcaR variants of (A) pSen5BFA4 (blue) and (C) pSen6MSA4 (brown) highlighting the location of the binding pocket (square) of the most suitable ligands of the respective biosensor variant (5BFA, cyan; 6MSA, navy). Substituted residues in comparison to the wild-type HcaR are shown in magenta. (B) Docking poses of 5BFA (cyan), CA (green), 4HCA (orange), 6MSA (navy), and PP (red) in the HcaR variant of pSen5BFA4. Except for PP, all ligands form hydrogen bonds to Y102. (D) Docking posesof 6MSA (navy), 4HBA (white), and $4 \mathrm{HCA}$ (orange) in the HcaR variant of pSen6MSA4. 6MSA is the only ligand able to form hydrogen bonds to N230 of the HcaR protein of pSen6MSA4. 


\section{References}

(1) Flachbart, L. K.; Sokolowsky, S.; Marienhagen, J. Displaced by Deceivers - Prevention of Biosensor Cross-Talk Is Pivotal for Successful Biosensor-Based High-Throughput Screening Campaigns. ACS Synth. Biol. 2019, 8 (8), acssynbio.9b00149. https://doi.org/10.1021/acssynbio.9b00149.

(2) Patrick, W. M.; Firth, A. E.; Blackburn, J. M. User-Friendly Algorithms for Estimating Completeness and Diversity in Randomized Protein-Encoding Libraries. Protein Eng. 2003, 16 (6), 451-457. 